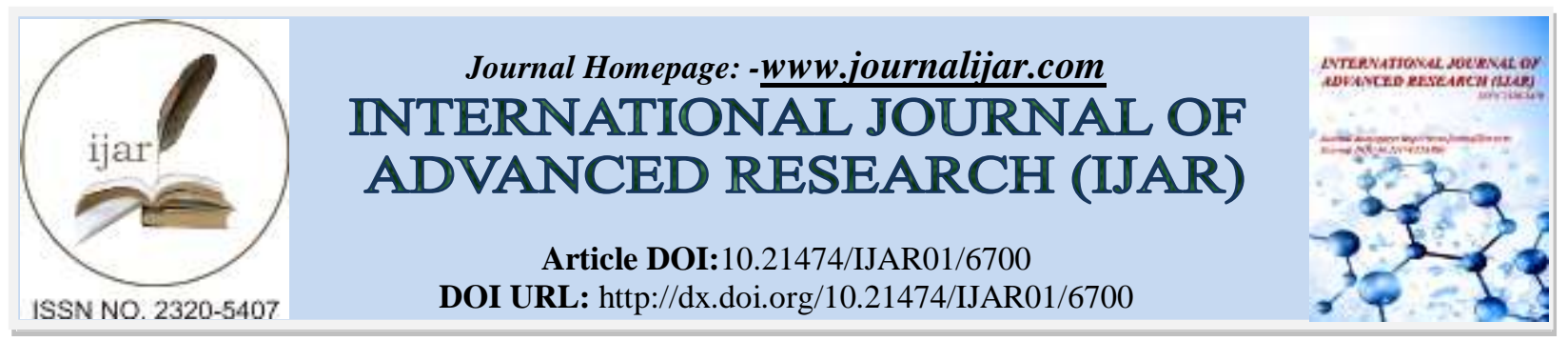

RESEARCH ARTICLE

\title{
GC-MS ANALYSIS OF BIOACTIVE COMPOUNDS AND ANTIMICROBIAL ACTIVITY OF CRYPTOCOCCUS RAJASTHANENSIS KY627764 ISOLATED FROM BOMBYX MORI GUT MICROFLORA.
}

Delicia Avilla Barretto and Shyam Kumar Vootla.

Department of Microbiology and Biotechnology, Karnatak University, Dharwad, Karnataka, India-580003.

\section{Manuscript Info}

...........................

Manuscript History

Received: 08 January 2018

Final Accepted: 10 February 2018

Published: March 2018

Keywords:-

Bioactive compounds - gut microflora 'Cryptococcus rajasthanensis $\cdot$ Bombyx mori.

\section{Abstract}

The insect gut microflora acts as a large reservoir of bioactive natural products as a diversity of resident microflora is found to be symbiotically associated with the insect. The present study reports the bioactive chemical compounds and the antimicrobial nature of the yeast isolated from the insect Bombyx mori gut. The yeast is identified as Cryptococcus rajasthanensis by Molecular characterization. The Fourier transform infrared spectroscopy (FT-IR) studies of the chloroform and ethyl acetate crude yeast extracts indicated a number of functional groups like alcoholic, phenolic, ester, aldehydic, etc. that accounts for the bioactive nature of the extracts. The Gas chromatography-Mass spectroscopy (GC-MS) analysis of the crude extracts revealed a large number of bioactive compounds of high and low molecular weight that are considered biologically active. Some of the biologically active molecules like phenol 2,4-bis $(1,1-$ dimethylethyl), 1,2-benzenedicarboxylic acid dibutyl ester, celidoniol deoxy, nonadecane, tetratetracontane, 2-methyloctacosane and pentadecane bearing antimicrobial property were detected in both chloroform and ethyl acetate extracts. Furthermore, the crude extracts were subjected to antimicrobial assay by agar well diffusion method and are found to be antimicrobial against four pathogenic bacteria Staphylococcus aureus, Bacillus subtilis, Escherichia coli and Pseudomonas aeruginosa and two fungi Candida albicans and Aspergillus flavus. The chloroform extract showed maximum inhibition for $E$. coli and minimum for A. flavus.

Copy Right, IJAR, 2018,. All rights reserved.

\section{Introduction:-}

Insects being one of the oldest group of animals on our planet that are ubiquitous in nature and have been adapted to several environmental conditions represent the most diverse group of animals on earth (Chapman, 2007). Thus, a wide range of microbes are found associated with these insects and are found to thrive on the insect's exoskeleton and in the hemocoel and gut of insects (Douglas et al., 2015). The prevalence of microbes in the insect gut is high as compared to the other parts of the insect anatomy as the gut tends to provide a hostile environment for the microorganisms. It is studied that the microbial load in the insect gut is 10 times the total cells of the insect (Rajagopal, 2009). The gut microbiota represent both positive as well as negative interaction 
with the host ranging from pathogenic to mutualistic relationships. Most of the microbes that live in association with the insect play beneficial roles for the host and hence play symbiotic or mutualistic role (Dillon and Dillon, 2004). The resident microbial flora in insects thus benefits the insect by producing essential compounds like vitamins, digesting and metabolizing food, nutrient absorption, etc. (Douglas et al., 2001; Nasir and Noda, 2003; Calderon-Cortes et al., 2012) and pheromones production and chemical communication (Ezenwa et al., 2012). The commensal flora are found to play crucial part in survival of the insects by protecting their insect hosts against the natural enemies and pathogens through varied mechanisms that include colonial resistance, production of toxins and insects immune system activation to fight the invader organism (Boulanger et al., 2001; Dillon and Charnley, 2002; 1995).

Although most studies in the past have focused on the bacterial endosymbionts, certain recent studies have now been conducted on the use of fungi, the yeasts being one of the predominant group as the beneficial microbes in the insect health (Gonzalez, 2014). Most of the yeasts insect endosymbionts are known to belong to the genus Candida, Metschnikowia, Pichia, Saccharomyces, Cryptococcus and Pseudozyma (Urubschurov and Janczyk, 2011). The yeast-insect symbiotic relationship benefits the yeasts as the insect provides hostile environment for the yeast to thrive in the gut, and facilitates dispersal of yeast spores and outbreeding (Vega and Dowd, 2005). On the contrary the insect is benefitted from the yeasts as the yeasts provide nutrients (vitamins like B3 and B5, trace metals, proteins, amino acids, etc.), detoxify harmful substances (usually by producing detoxifying enzymes like hydrolase, glucosidase, etc.), provides protection from biotic and abiotic stress (by providing protection from invader pathogens) and can also aid in chemical communication (Gibson and Hunter, 2010; Engel and Moran, 2013; Pozo et al., 2012; Davis et al., 2011; Christensen, 2010). This insect-microbe association directs the research onto a wide range of novel chemical moieties that can be obtained from the microorganism isolated from the insect gut that can be used in human therapy (Beemelmanns et al., 2016; Brachmann and Bode, 2013). The research on the secondary metabolites produced by the gut microflora can be justified in 2 ways: 1) to identify the natural products produced by the microflora that can be beneficial to humans and 2) to identify the known as well as novel microorganisms involved in production of these natural compounds so as to exploit these microbes as they may produce these secondary metabolites to combat pathogenic microbes (Soria-Mercado et al., 2012).

The insect Bombyx mori (B. mori) belongs to the Lepidopteran class of insects wherein the yeast association with the insect has been less well studied (Gonzalez, 2014). Although the yeasts are known to produce volatile compounds that can be used as biocontrol agents especially in post harvest crop management (Witzgall et al., 2012), very less research has been conducted for use of the bioactive compounds produced by the yeasts isolated from the insect gut that can be used in human therapy (Bode, 2011). Therefore, the present study was focused on the investigation of antimicrobial nature of the yeast isolated from the B. mori gut and evaluation of its bioactive chemical constituents by the GC-MS analysis. Our studies have successively proved that the yeast Cryptococcus rajasthanensis isolated from the $B$. mori gut bears antimicrobial potential and the GC-MS profiling has shown a wide range of chemical compounds bearing therapeutic properties including antimicrobial, antioxidant, anticancer, etc.

\section{Materials and Methods:- \\ Isolation of yeast from Bombyx mori gut:-}

Fifth instar healthy B. mori larva was dissected under sterile conditions, the gut was isolated and washed in sterile distilled water to remove solid contaminants. The fluid was collected after slight maceration of the gut in sterile bacteriological saline. The extracted fluid containing microorganisms was swab inoculated onto the sterile potato dextrose agar (PDA) plate. The inoculated plate was incubated for $48-72$ hours at $27-30{ }^{\circ} \mathrm{C}$.

\section{Identification of the yeast strain:-}

The isolated yeast Bm5F5 strain was initially checked for its morphological characteristics and gram stained. It was further identified by molecular characterization using $18 \mathrm{~S}$ rRNA gene sequencing and phylogenetic analyses.

\section{Preparation of yeast extracts:-}

The yeast extracts were prepared according to Cita et al., 2017. Briefly, the yeast from the PDA plate was inoculated into an Erlenmeyer flask containing $500 \mathrm{ml}$ sterile saboraud's broth. The inoculated flask was incubated at $27^{\circ} \mathrm{C}$ for 5-7 days under shaker conditions. After incubation, the culture broth was filtered to remove the cell mass. The filtrate was extracted with chloroform as well as ethyl acetate $(1: 1 \mathrm{v} / \mathrm{v})$. The solvent extract was concentrated by rota-evaporation. The chloroform and ethyl acetate crude extracts of the yeast was used for further evaluation. 
Fourier transform infrared radiation (FTIR) analysis of yeast extracts:-

The various functional groups responsible for the biological activities of the crude yeast extracts were recorded (Poojary et al., 2015). KBr pellets of the dried yeast extracts were prepared and the spectra were recorded ranging from $4000-400 \mathrm{~cm}^{-1}$ at a resolution of $2 \mathrm{~cm}^{-1}$ using a FTIR spectrophotometer (NICOLET 6700, USA).

\section{Gas chromatography-Mass spectroscopy analysis of yeast extracts:-}

The GC-MS analysis of the crude yeast extracts was carried out to evaluate the various bioactive compounds present in the yeast extracts (Sivaraman et al., 2017). This was carried out by GC-MS spectroscopy (Shimadzu GCMS-QP 2010S system) and the compounds were predicted using the National Institute Standard and Technology 11.0 (NIST) library database and Wiley 8.0.

\section{Evaluation of Antimicrobial activity by agar well diffusionassay:-}

The antimicrobial activities of the yeast extracts were performed by agar well diffusion method (Bonev et al., 2008). Lag phase cultures of gram positive bacteria Staphylococcus aureus and Bacillus subtilis, gram negative bacteria Escherichia coli and Pseudomonas aeruginosa and fungi Candida albicans and Aspergillus flavus were used as the test microorganisms. $100 \mu \mathrm{l}$ of the test microorganisms were swab inoculated onto the sterile nutrient agar and sterile PDA petriplates respectively for which then wells were bored. $50 \mu \mathrm{g} / \mathrm{mL}$ of the yeast extracts were added into the respectively labeled well. Tetracycline and Streptomycin were used as the reference drugs for the bacteria and Nystatin and fluconazole for fungi. These plates were incubated at $37^{\circ} \mathrm{C}$ for 24 hours for bacteria and $27-30^{\circ} \mathrm{C}$ for 48 hours for fungi followed by observing and measuring the antimicrobial activity of the yeast extracts by measuring the zones of inhibition in millimeters using the Hi media Antibiotic scale.

\section{Results:-}

Isolation of yeast from Bombyx mori gut:-

A cream colored glistening yeast colony was isolated on potato dextrose agar as shown in figure 1 and labeled as yeast Bm5F5 strain for reference.

\section{Identification of the yeast strain:-}

The yeast Bm5F5 strain depicted encapsulated oval shaped cells on gram staining as in figure 2. The strain was identified as Cryptococcus rajasthanensis by $18 \mathrm{~S}$ rRNA gene sequencing and phylogenetic analyses and the gene sequence was deposited in the NCBI GenBank under the accession number KY627764.

Fourier transform infrared radiation (FTIR) analysis of yeast extracts:-

FT-IR spectral data of the chloroform and ethyl acetate crude extracts revealed presence of various functional groups in the yeast as shown in figure 3 and 4 respectively. Spectral data in table 1 gives the interpretation of the frequencies, vibration bonds and the functional groups present in the extracts. Both the extracts show hydrogen bonded $-\mathrm{OH}$ stretching, $\mathrm{C}-\mathrm{H}$ stretching, $\mathrm{N}-\mathrm{H}$ bending, $\mathrm{C}-\mathrm{C}$ stretching in ring, $\mathrm{C}-\mathrm{O}$ stretching and $\mathrm{C}-\mathrm{N}$ stretching in common.

Gas chromatography-Mass spectroscopy analysis of yeast extracts:-

The bioactive compounds present in chloroform and ethyl acetate extracts obtained from the yeast are shown in tables 2 and 3. The GC-MS studies of the yeast extracts showed 18 compounds in the chloroform extract and 15 compounds in the ethyl acetate extract. Based on the relative abundance, the top four major compounds present in the chloroform extract were Celidoniol deoxy (23.71\%), eicosane (22.26\%), nonadecane (18.68\%) and tetratetracontane (15.93\%). The ethyl acetate extract contained 2-methyloctacosane (16.37\%), nonadecane (15.03\%), Celidoniol, deoxy (13.64\%) and tetratetracontane (10.43\%) as the top four major compounds. The GC chromatogram of the chloroform and ethyl acetate extracts are depicted in figures 5 and 6 respectively.

\section{Evaluation of Antimicrobial activity by agar well method:-}

A concentration of $50 \mu \mathrm{g} / \mathrm{mL}$ of the yeast extracts was evaluated for its antimicrobial activity against 4 bacterial and 2 fungal strains. The results shown in table 4 and figure 7 determine the chloroform and ethyl acetate extracts of the yeast as antimicrobial agents against human pathogens. It has shown good antibacterial activity against the gram negative bacterial strains used Escherichia coli and Pseudomonas aeruginosa as well as to the gram positive ones Staphylococcus aureus and Bacillus subtilis. The antifungal activity of the yeast extracts against the yeast Candida albicans and the fungal strain Aspergillus flavus predicts the antifungal nature of the extracts. 
Fig. 1:- Cream colored glistening yeast colony on Potato Dextrose agar isolated from Bombyx mori gut

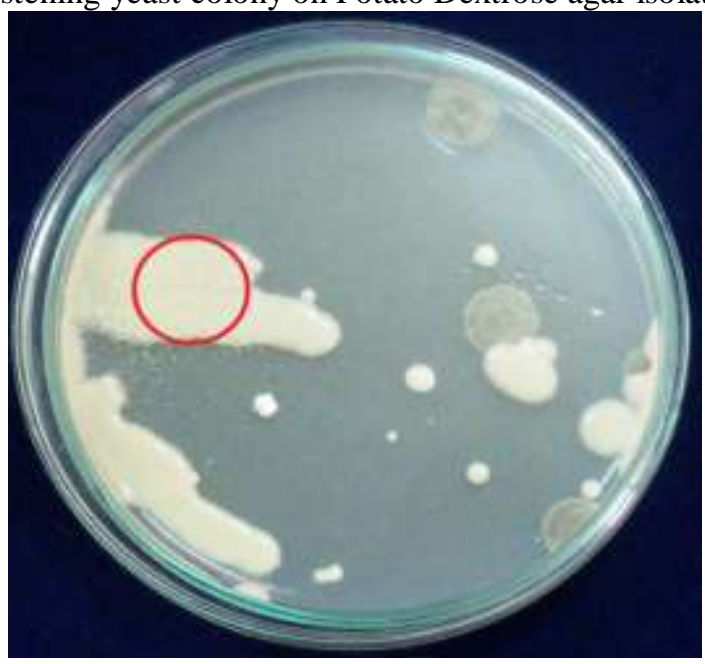

Fig. 2:- Gram staining showing gram positive encapsulated oval shaped cells

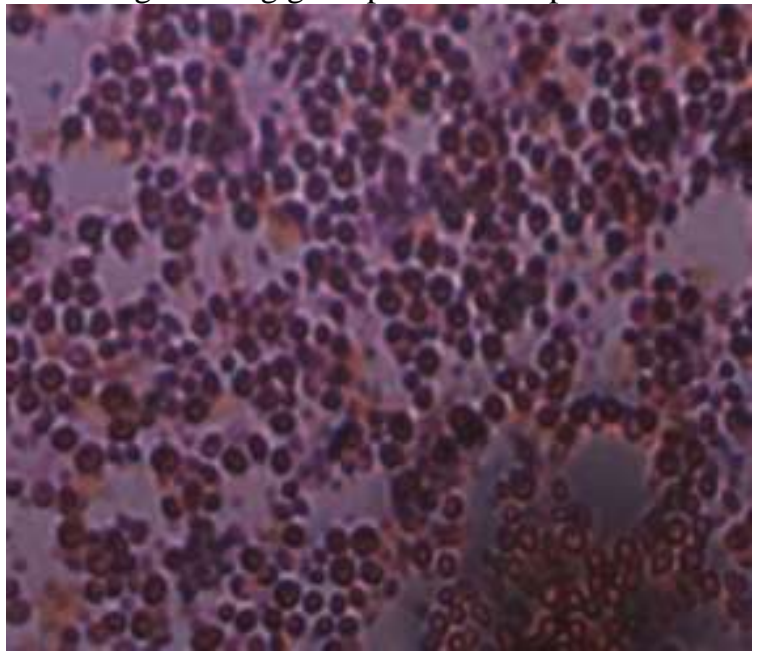

Fig. 3:- FT-IR spectrum of the yeast Cryptococcus rajasthanensis chloroform extract

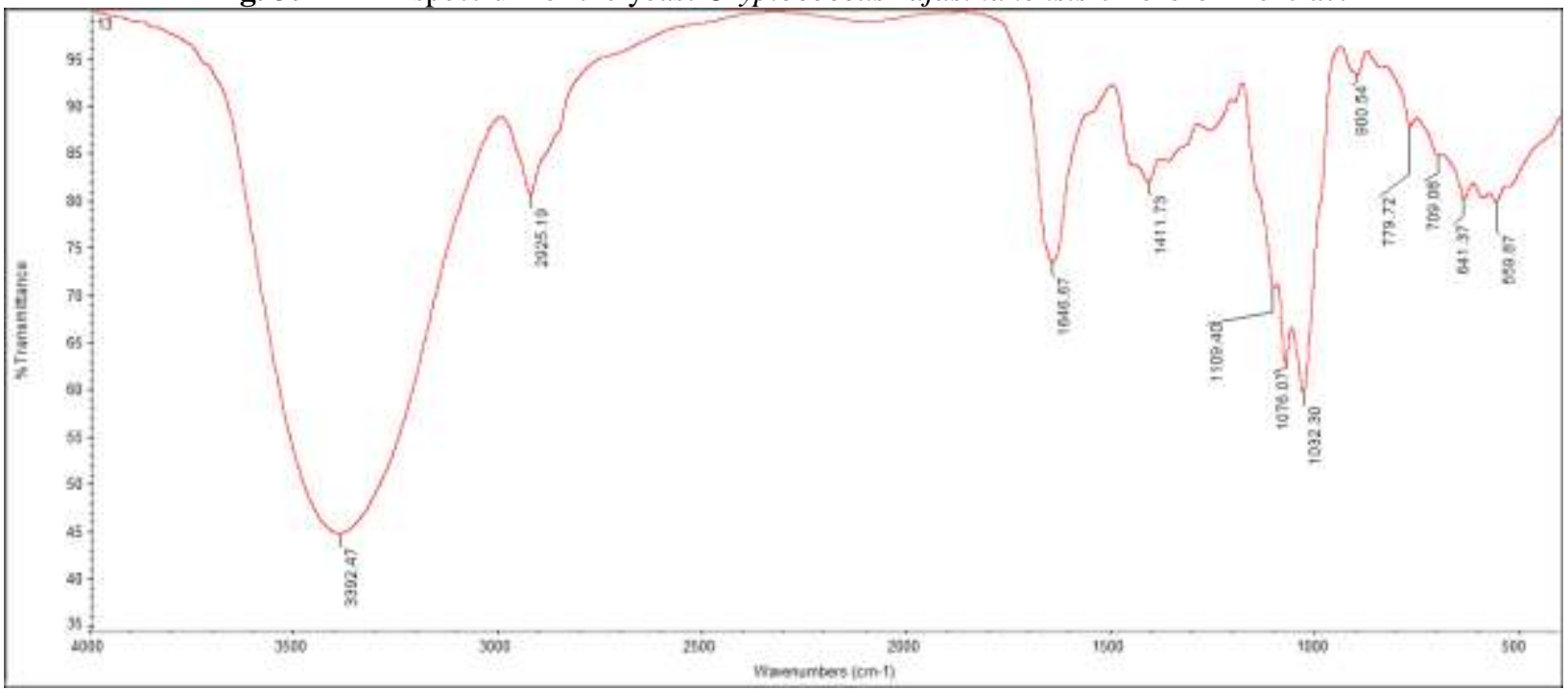


Fig. 4:- FT-IR spectrum of the yeast Cryptococcus rajasthanensis ethyl acetate extract

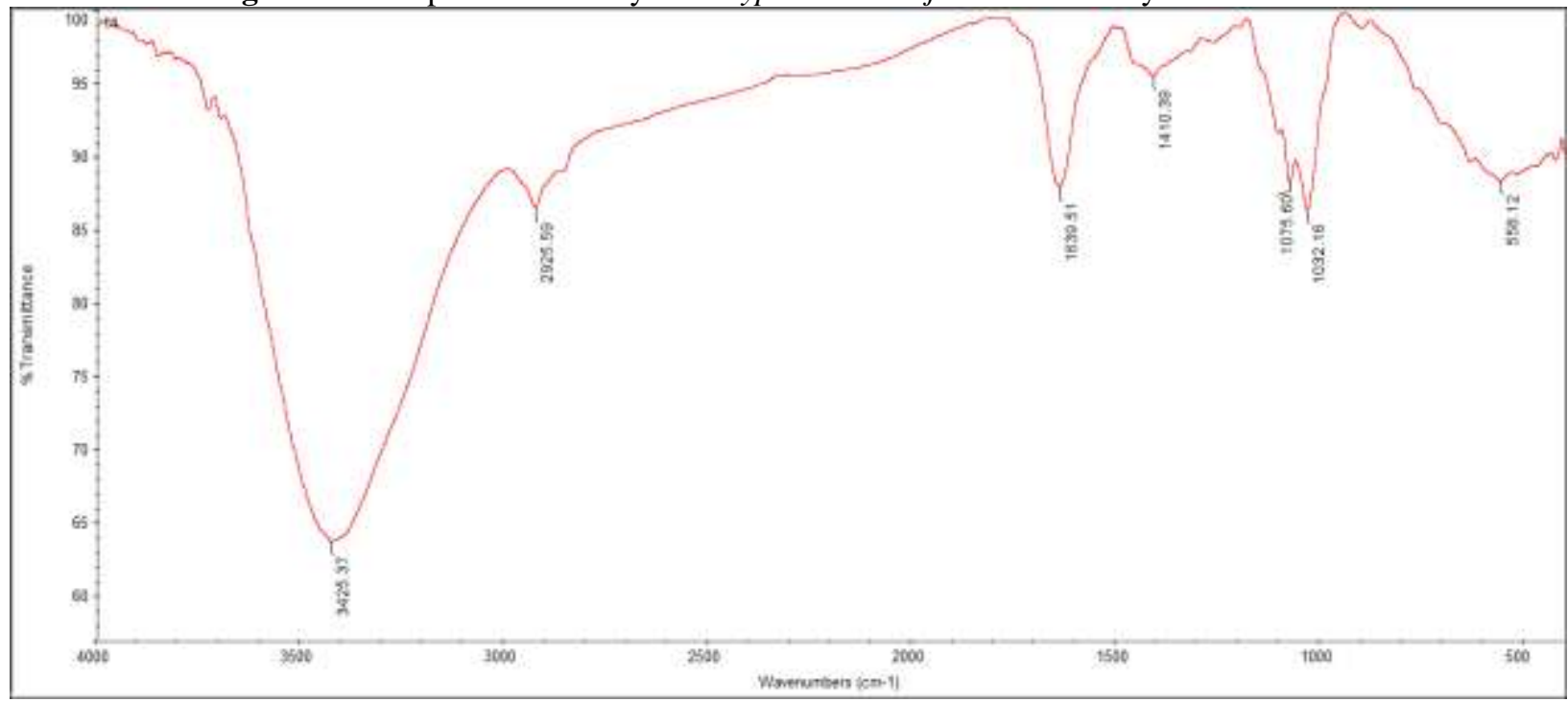

Fig.5:- GC-MS chromatogram of the yeast Cryptococcus rajasthanensis chloroform extract

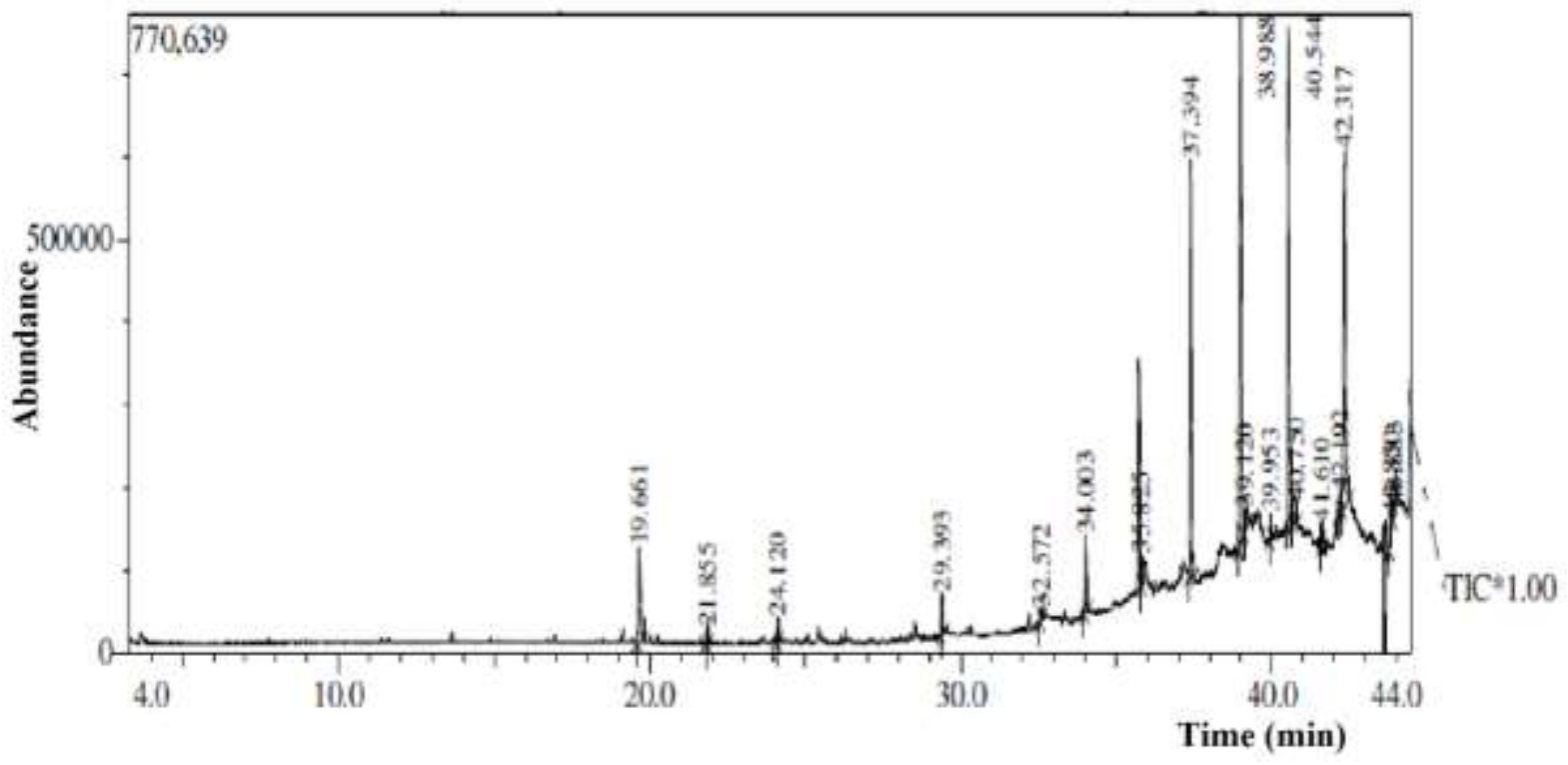


Fig. 6:-GC-MS chromatogram of the yeast Cryptococcus rajasthanensis ethyl acetate extract

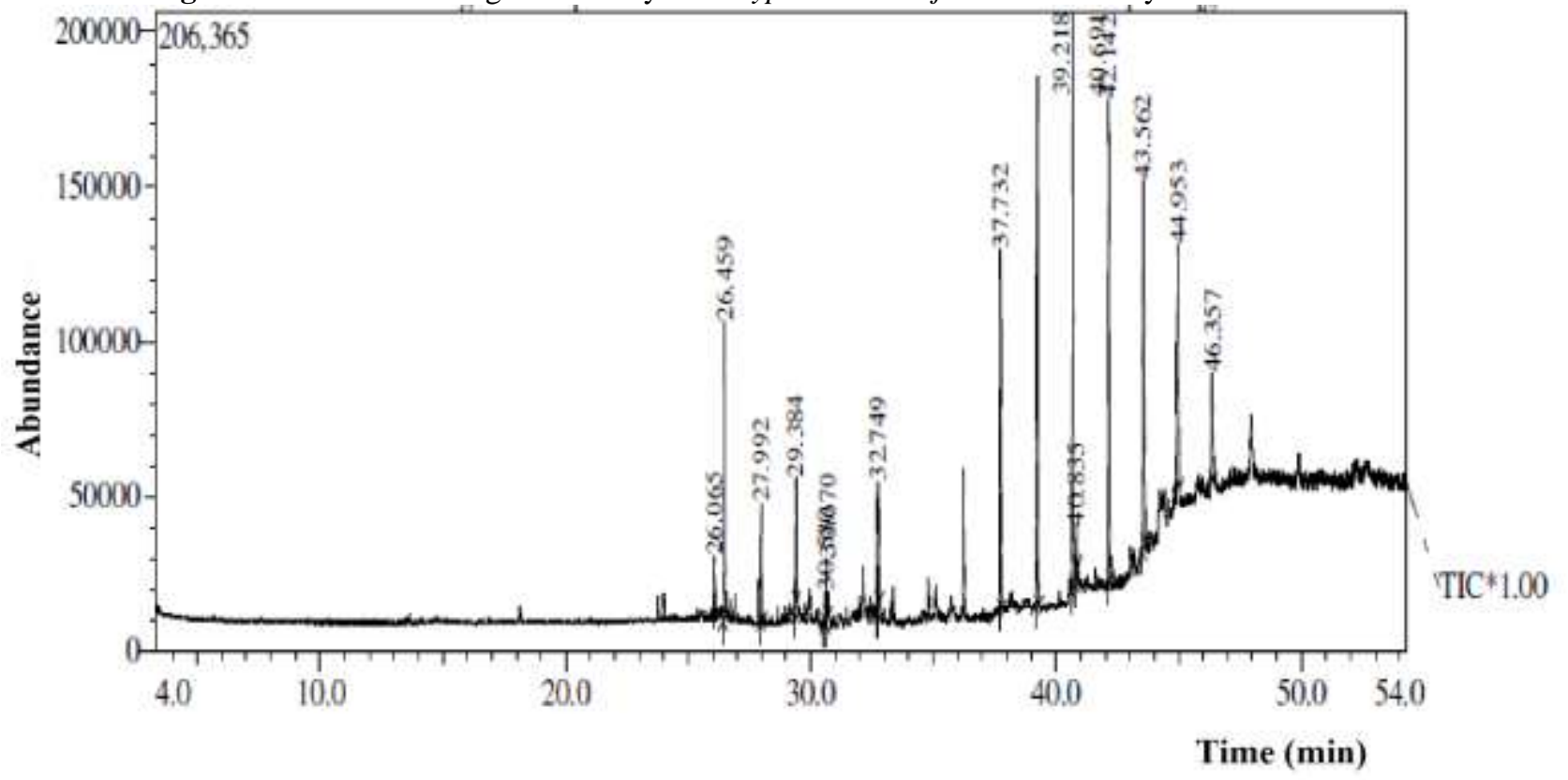

Fig. 7:- Antimicrobial activity of yeast Cryptococcus rajasthanensis extracts against human pathogens by agar well
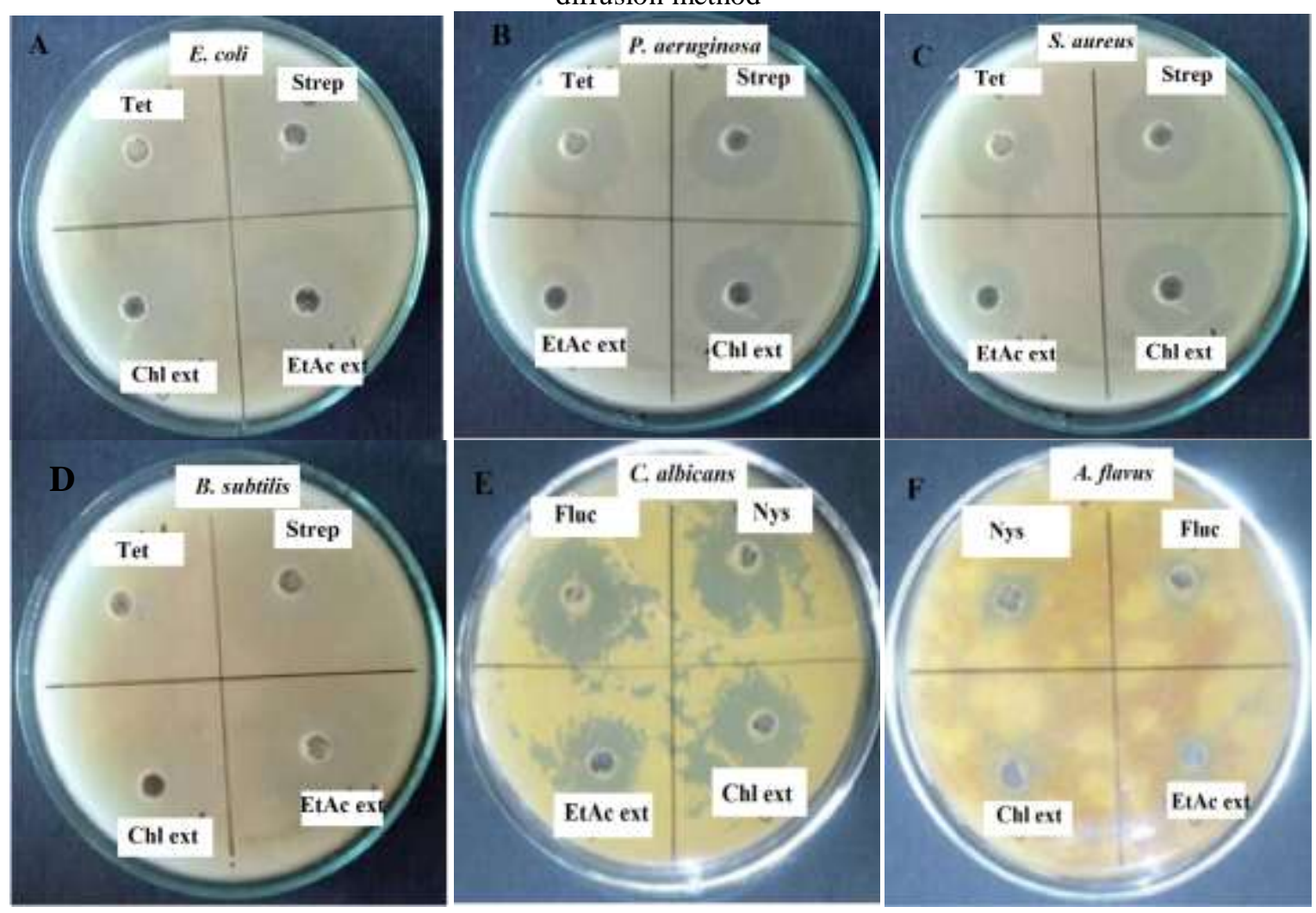

Note:- Tet-Tetracycline; Strep- Streptomycin; Fluc- Fluconazole; Nys- Nystatin; Chl ext- Chloroform extract; EtAc ext- Ethyl acetate extract 


\begin{tabular}{|c|c|c|}
\hline IR $v_{\max }\left(\mathrm{cm}^{-1}\right)$ & (Vibration mode) & Functional groups \\
\hline \multicolumn{3}{|l|}{ Chloroform extract } \\
\hline \multirow[t]{2}{*}{3392.47} & H-bonded OH-stretching; & Alcohols, phenols \\
\hline & $-\mathrm{N}-\mathrm{H}$ stretching & $1^{\circ}, 2^{\circ}$ amines, amides \\
\hline 2925.19 & C-H stretching & Alkanes \\
\hline 1646.67 & $-\mathrm{C}=\mathrm{C}-$ stretching & alkenes \\
\hline 1411.73 & C-C stretch (in-ring) & aromatics \\
\hline \multirow{2}{*}{$\begin{array}{ll}1109.40, & 1076.07 \\
1032.30 & \end{array}$} & -C-O stretching; & Alcohols, carboxylic acid, esters, ethers; \\
\hline & -C-N stretching & Aliphatic amines \\
\hline 900.54 & $=\mathrm{C}-\mathrm{H}$ bending & alkenes \\
\hline \multirow[t]{3}{*}{$779.72 ; 709.08$} & N-H waging & 1, 20 amines; \\
\hline & C-H “oop” & Aromatics; \\
\hline & $\mathrm{C}-\mathrm{Cl}$ stretching & Alkyl halides \\
\hline 641.37 & $-\mathrm{C} \equiv \mathrm{C}-\mathrm{H}: \mathrm{C}-\mathrm{H}$ bending & alkynes \\
\hline 559.87 & C-Br stretching & Alkyl halides \\
\hline \multicolumn{3}{|l|}{ Ethyl acetate extract } \\
\hline 3425.37 & H-bonded OH-stretching & Alcohols, phenols \\
\hline 2925.59 & $\mathrm{C}-\mathrm{H}$ stretching & Alkanes \\
\hline 1639.51 & $\mathrm{~N}-\mathrm{H}$ bending & 1, 20 amines \\
\hline 1410.39 & C-C stretch (in-ring) & aromatics \\
\hline $1075.60 ; 1032.16$ & $\begin{array}{l}\text { C-O stretching; } \\
\text { C-N stretching }\end{array}$ & $\begin{array}{l}\text { Alcohols, carboxylic acids, esters, ethers; } \\
\text { Aliphatic amines }\end{array}$ \\
\hline 558.12 & C-Br stretching & Alkyl halides \\
\hline
\end{tabular}

Table 1:- FTIR data for the yeast Cryptococcus rajasthanensis extracts 


\begin{tabular}{|c|c|c|c|c|c|c|}
\hline $\begin{array}{l}\text { Sl } \\
\text { no }\end{array}$ & Compound name & $\begin{array}{l}\text { Reten- } \\
\text { tion } \\
\text { time }\end{array}$ & $\begin{array}{l}\text { Peak } \\
\text { Area } \\
(\%)\end{array}$ & $\begin{array}{l}\text { Molecular } \\
\text { formula and } \\
\text { Molecular } \\
\text { weight }\end{array}$ & Activity & Reference \\
\hline 1 & $\begin{array}{l}\text { Phenol-2,4-Bis(1,1- } \\
\text { dimethylethyl)- }\end{array}$ & 19.661 & 4.42 & 206 & $\begin{array}{l}\text { Antioxidant (Inhibits } \\
\text { ROS) } \\
\text { Antibacterial }\end{array}$ & $\begin{array}{l}\text { Teresa et al., } 2014 \\
\text { Manorenjitha et al., } \\
2013\end{array}$ \\
\hline 2 & Pentadecane & 21.855 & 0.49 & $212\left(\mathrm{C}_{15} \mathrm{H}_{32}\right)$ & Antibacterial & $\begin{array}{l}\text { Yogeshwari et al., } \\
2012\end{array}$ \\
\hline 3 & Heptane,3,3-dimethyl- & 24.120 & 0.78 & $128.25\left(\mathrm{C}_{9} \mathrm{H}_{20}\right)$ & Unknown & \\
\hline 4 & $\begin{array}{l}\text { 1,2-Benzenedicarboxylic } \\
\text { acid, dibutyl ester } \\
\text { (Dibutyl phthalate) }\end{array}$ & 29.393 & 1.51 & $\begin{array}{l}278.344 \\
\left(\mathrm{C}_{15} \mathrm{H}_{28} \mathrm{O}\right)\end{array}$ & $\begin{array}{l}\text { Antibacterial } \\
\text { Antifouling } \\
\text { Pesticide }\end{array}$ & $\begin{array}{l}\text { Khatiwora et al., } \\
2012 \\
\text { Jenecius et al., } \\
2012 \\
\text { Wanxi et al., } 2014\end{array}$ \\
\hline 5 & 3-Ethyl-3-methylheptane & 32.572 & 0.40 & $142.28\left(\mathrm{C}_{14} \mathrm{H}_{22}\right)$ & Unknown & \\
\hline 6 & 2-methyloctacosane & 34.003 & 2.75 & $\begin{array}{l}408.7867 \\
\left(\mathrm{C}_{29} \mathrm{H}_{60}\right)\end{array}$ & $\begin{array}{l}\text { Found in cuticles of } \\
\text { various insects spp. } \\
\text { (chemical } \\
\text { communication) }\end{array}$ & Spikes et al., 2010 \\
\hline 7 & $\begin{array}{l}\text { 2-Cyclohexyl-3-isopropyl- } \\
\text { pent-4-en-2-ol }\end{array}$ & 35.825 & 0.94 & $\begin{array}{l}210.356 \\
\left(\mathrm{C}_{14} \mathrm{H}_{26} \mathrm{O}\right)\end{array}$ & unknown & \\
\hline 8 & Nonadecane & 37.394 & 16.37 & $268\left(\mathrm{C}_{19} \mathrm{H}_{40}\right)$ & $\begin{array}{l}\text { Antimicrobial and } \\
\text { cytotoxic } \\
\text { A cuticular } \\
\text { hydrocarbon of } \\
\text { insects (chemical } \\
\text { communication) }\end{array}$ & $\begin{array}{l}\text { Hsouna et al., } 2011 \\
\text { Colazza et al., } 2007\end{array}$ \\
\hline 9 & Eicosane & 40.544 & 22.11 & $\begin{array}{l}282.547 \\
\left(\mathrm{C}_{20} \mathrm{H}_{42}\right)\end{array}$ & Antitumor activity & $\begin{array}{l}\text { Sivasubramanian } \\
\text { and Brindha, } 2013\end{array}$ \\
\hline 10 & $\begin{array}{l}\text { 2,5-Octadecanoic acid, } \\
\text { methyl ester }\end{array}$ & 39.120 & 1.35 & $\begin{array}{l}408.7867 \\
\left(\mathrm{C}_{19} \mathrm{H}_{30} \mathrm{O}_{2}\right)\end{array}$ & $\begin{array}{l}\text { Antiviral } \\
\text { Antioxidant, } \\
\text { antibacterial }\end{array}$ & $\begin{array}{l}\text { Linton et al., } 2013 \\
\text { Sudharshan et al., } \\
2010\end{array}$ \\
\hline 11 & 2-Methyltetracosane & 39.953 & 0.87 & $\begin{array}{l}352.680 \\
\left(\mathrm{C}_{25} \mathrm{H}_{52}\right)\end{array}$ & $\begin{array}{l}\text { Free radical } \\
\text { scavenging activity }\end{array}$ & Ramya et al., 2015 \\
\hline 12 & Hexatriacontane & 41.610 & 1.26 & $\begin{array}{l}506.973 \\
\left(\mathrm{C}_{36} \mathrm{H}_{74}\right) \\
\end{array}$ & Unknown & \\
\hline 13 & $\begin{array}{l}\text { Celidoniol, deoxy } \\
\text { (Nonacosane) }\end{array}$ & 38.968 & 21.11 & $\begin{array}{l}408 \\
\left(\mathrm{C}_{29} \mathrm{H}_{60}\right)\end{array}$ & $\begin{array}{l}\text { Antibacterial } \\
\text { Antiinflammatory } \\
\text { Chemical } \\
\text { communication } \\
\text { especially in } \\
\text { Anopheles stephensi } \\
\text { mosquito } \\
\text { Pheromone of Orgyia } \\
\text { leucostigma }\end{array}$ & $\begin{array}{l}\text { Kose et al., } 2016 \\
\text { Zakaria et al., } 2014 \\
\text { Brei et al., } 2004\end{array}$ \\
\hline
\end{tabular}




\begin{tabular}{|c|c|c|c|c|c|c|}
\hline 14 & 2-Bromotetradecane & 40.750 & 2.17 & $\begin{array}{l}277.284 \\
\left(\mathrm{C}_{14} \mathrm{H}_{29} \mathrm{Br}\right)\end{array}$ & Unknown & \\
\hline 15 & Methenolone & 42.192 & 1.25 & $\begin{array}{l}302.451 \\
\left(\mathrm{C}_{20} \mathrm{H}_{30} \mathrm{O}_{2}\right)\end{array}$ & $\begin{array}{l}\text { A naturally occurring } \\
\text { drug for aplastic } \\
\text { anemia treatment }\end{array}$ & Lockner, 1979 \\
\hline 16 & Tetratetracontane & 42.317 & 21.18 & $\begin{array}{l}619.185 \\
\left(\mathrm{C}_{44} \mathrm{H}_{90}\right)\end{array}$ & Antibacterial & $\begin{array}{l}\text { Gumgumjee and } \\
\text { Hajar, 2015 }\end{array}$ \\
\hline 17 & 1-Chlorononadecane & 43.850 & 0.60 & $\begin{array}{l}302.966 \\
\left(\mathrm{C}_{19} \mathrm{H}_{39} \mathrm{Cl}\right)\end{array}$ & Unknown & \\
\hline 18 & $\begin{array}{l}\text { 3-Phenoxypropylamine,2- } \\
\text { allyl-,beta,hydroxyl-N- } \\
\text { [3.3-dimethylpropargyl]- }\end{array}$ & 43.883 & 0.43 & & Unknown & \\
\hline
\end{tabular}

Table 2:- Chemical constituents of the yeast Cryptococcus rajasthanensis chloroform extract obtained by GC-MS analysis

\begin{tabular}{|c|c|c|c|c|c|c|}
\hline $\begin{array}{l}\text { Sl } \\
\text { no }\end{array}$ & Compound name & $\begin{array}{l}\text { Reten- } \\
\text { tion } \\
\text { time }\end{array}$ & $\begin{array}{l}\text { Peak } \\
\text { Area } \\
(\%)\end{array}$ & $\begin{array}{l}\text { Molecular } \\
\text { formula and } \\
\text { Molecular } \\
\text { weight }\end{array}$ & Activity & Reference \\
\hline & Pentadecane & 26.065 & 1.05 & $212\left(\mathrm{C}_{15} \mathrm{H}_{32}\right)$ & Antibacterial & $\begin{array}{l}\text { Yogeshwari et al., } \\
2012\end{array}$ \\
\hline & $\begin{array}{l}\text { Phenol-2,4-Bis(1,1- } \\
\text { Dimethylethyl)- }\end{array}$ & 26.459 & 8.16 & $\begin{array}{l}206 \\
\left(\mathrm{C}_{14} \mathrm{H}_{22} \mathrm{O}\right)\end{array}$ & $\begin{array}{l}\text { Antioxidant } \\
\text { Antibacterial }\end{array}$ & $\begin{array}{l}\text { Teresa et al., } 2014 \\
\text { Manorenjitha et al., } \\
2013\end{array}$ \\
\hline & Tetradecane & 27.992 & 2.02 & & Unknown & \\
\hline & Octadecane & 29.384 & 1.99 & $\begin{array}{l}254.494 \\
\left(\mathrm{C}_{18} \mathrm{H}_{38}\right)\end{array}$ & $\begin{array}{l}\text { Antifungal agent for } \\
\text { plant and human } \\
\text { pathogens }\end{array}$ & $\begin{array}{l}\text { Abubacker and } \\
\text { Devi, } 2015\end{array}$ \\
\hline & $\begin{array}{l}\text { Bis-(3,5,5-trimethylhexyl) } \\
\text { ether }\end{array}$ & 30.580 & 0.52 & & Unknown & \\
\hline & Docosane & 30.670 & 1.22 & $\begin{array}{l}310.60064 \\
\left(\mathrm{C}_{22} \mathrm{H}_{46}\right)\end{array}$ & $\begin{array}{l}\text { Antibacterial activity } \\
\text { Enhances host egg } \\
\text { parasitization }\end{array}$ & $\begin{array}{l}\text { Gumgumjee and } \\
\text { Hajar, } 2015 \\
\text { Paul et al., } 2002\end{array}$ \\
\hline & $\begin{array}{l}\text { 1,2-Benzenedicarboxylic } \\
\text { acid, dibutyl ester }\end{array}$ & 32.749 & 3.10 & $\begin{array}{l}278.344 \\
\left(\mathrm{C}_{15} \mathrm{H}_{28} \mathrm{O}\right)\end{array}$ & $\begin{array}{l}\text { Antibacterial } \\
\text { Antifouling } \\
\text { Pesticide }\end{array}$ & $\begin{array}{l}\text { Khatiwora et al., } \\
2012 \\
\text { Jenecius et al., } 2012 \\
\text { Wanxi et al., } 2014\end{array}$ \\
\hline & Tetracosane & 37.732 & 9.65 & $\begin{array}{l}338.66 \\
\left(\mathrm{C}_{24} \mathrm{H}_{50}\right)\end{array}$ & $\begin{array}{l}\text { Cytotoxic towards } \\
\text { gastric cancer cells by } \\
\text { induction of apoptosis }\end{array}$ & Uddin et al., 2012 \\
\hline & Nonadecane & 39.218 & 15.64 & $\begin{array}{l}268 \\
\left(\mathrm{C}_{19} \mathrm{H}_{40}\right)\end{array}$ & $\begin{array}{l}\text { Antimicrobial and } \\
\text { cytotoxic } \\
\text { A cuticular } \\
\text { hydrocarbon of insects } \\
\text { (chemical } \\
\text { communication) }\end{array}$ & $\begin{array}{l}\text { Hsouna et al., } 2011 \\
\text { Colazza et al., } 2007\end{array}$ \\
\hline & 2-methyloctacosane & 40.691 & 17.48 & $\begin{array}{l}408.7867 \\
\left(\mathrm{C}_{29} \mathrm{H}_{60}\right)\end{array}$ & $\begin{array}{l}\text { Found in cuticles of } \\
\text { various insects spp. } \\
\text { (chemical }\end{array}$ & Spikes et al., 2010 \\
\hline
\end{tabular}




\begin{tabular}{|c|c|c|c|c|c|}
\hline & & & & communication) & \\
\hline $\begin{array}{l}\text { 1-Heptanol,2,4-dimethyl-, } \\
(\mathrm{R}, \mathrm{R})-(+)-\end{array}$ & 40.835 & 1.37 & & Unknown & \\
\hline $\begin{array}{l}\text { Celidoniol, deoxy } \\
\text { (Nonacosane) }\end{array}$ & 42.142 & 15.06 & $408\left(\mathrm{C}_{29} \mathrm{H}_{60}\right)$ & $\begin{array}{l}\text { Antibacterial } \\
\text { Antiinflammatory } \\
\text { Chemical } \\
\text { communication } \\
\text { especially in } \\
\text { Anopheles stephensi } \\
\text { mosquito } \\
\text { Pheromone of Orgyia } \\
\text { leucostigma }\end{array}$ & $\begin{array}{l}\text { Kose et al., } 2016 \\
\text { Zakaria et al., } 2014 \\
\text { Brei et al., } 2004 \\
\text { Grant et al., } 1987\end{array}$ \\
\hline Tetratetracontane & 43.562 & 11.16 & $\begin{array}{l}619.185 \\
\left(\mathrm{C}_{44} \mathrm{H}_{90}\right) \\
\end{array}$ & Antibacterial & $\begin{array}{ll}\text { Gumgumjee } & \text { and } \\
\text { Hajar, 2015 } & \\
\end{array}$ \\
\hline $\begin{array}{l}\text { Heptadecane, 2,6,10,15- } \\
\text { tetramethyl- }\end{array}$ & 44.953 & 8.16 & $296\left(\mathrm{C}_{21} \mathrm{H}_{44}\right)$ & Unknown & \\
\hline Decane,2,3,8-trimethyl- & 46.357 & 3.41 & & Unknown & \\
\hline
\end{tabular}

Table 3:- Chemical constituents of the yeast Cryptococcus rajasthanensis ethyl acetate extract obtained by GC-MS analysis

\begin{tabular}{|c|c|c|c|c|}
\hline \multicolumn{5}{|c|}{ Zones of inhibition in $\mathrm{mm}$} \\
\hline Test microorganism & $\begin{array}{c}\text { Chloroform } \\
\text { extract } \\
(50 \mu \mathrm{g} / \mathrm{mL})\end{array}$ & $\begin{array}{c}\text { Ethyl acetate } \\
\text { extract } \\
(50 \mu \mathrm{g} / \mathrm{mL})\end{array}$ & $\begin{array}{c}\text { Tetracycline } \\
(50 \mu \mathrm{g} / \mathrm{mL})\end{array}$ & $\begin{array}{c}\text { Streptomycin } \\
(50 \mu \mathrm{g} / \mathrm{mL})\end{array}$ \\
\hline \multicolumn{5}{|l|}{ Antibacterial activity } \\
\hline Escherichia coli & 17 & 16 & 20 & 19 \\
\hline Pseudomonas aeruginosa & 14 & 13 & 18 & 18 \\
\hline Staphylococcus aureus & 13 & 12 & 22 & 20 \\
\hline Bacillus subtilis & 15 & 14 & 23 & 21 \\
\hline \multicolumn{5}{|l|}{ Antifungal activity } \\
\hline Test microorganism & $\begin{array}{c}\text { Chloroform } \\
\text { extract } \\
(50 \mu \mathrm{g} / \mathrm{mL})\end{array}$ & $\begin{array}{c}\text { Ethyl acetate } \\
\text { extract } \\
(50 \mu \mathrm{g} / \mathrm{mL})\end{array}$ & $\begin{array}{c}\text { Nystatin } \\
(50 \mu \mathrm{g} / \mathrm{mL})\end{array}$ & $\begin{array}{c}\text { Fluconazole } \\
(50 \mu \mathrm{g} / \mathrm{mL})\end{array}$ \\
\hline Candida albicans & 18 & 10 & 16 & 20 \\
\hline Aspergillus flavus & 10 & 10 & 14 & 12 \\
\hline
\end{tabular}

Table 4:- Antimicrobial activity of yeast Cryptococcus rajasthanensis extracts against pathogens by Agar well diffusion assay

\section{Discussion:-}

The insect gut microflora has become a focus of intense research in recent years owing to the useful natural products that can be derived from the insect-microbe association that can be used for human therapy (Douglas, 2015). Likewise in the present study, a potent yeast strain was isolated from the Bombyx mori gut and was identified as Cryptococcus rajasthanensis. A research by Saluja and Prasad (2007) has first reported Cryptococcus rajasthanensis strain from Rajasthan, India from the Andrographis echioides inflorescence. Later on, it has also been isolated and studied in different sources like the phylloplane of rice, sugarcane, etc. (Limtong et al., 2014). The Cryptococcus spp. have also been reported from many insect orders including the Lepidopteran larval gut (Vega and Dowd, 2005). The yeast grew as cream colored glistening colony on potato dextrose agar which on gram staining appeared as gram positive oval shaped cells with capsules surrounding the yeast cells which is the peculiar characteristics of the Cryptococcal strain (Botton, 1980).

The FT-IR spectral analysis of the chloroform and ethyl acetate yeast extract depicted that the yeast extracts contain various biologically active functional groups like the alcoholic, phenolic, ester, aldehydic, etc. and hence proves that 
the yeast possesses bioactive chemical compounds (Poojary et al., 2015). FT-IR spectra of both the extracts showed peaks at $3392.47 \mathrm{~cm}^{-1}$ and $3425.37 \mathrm{~cm}^{-1}$ which could be due to the $\mathrm{OH}$ functional group present in the samples (Tejado et al., 2007). Although the functional groups present in the extracts responsible for the bioactive nature of the compounds can be predicted by the FT-IR analysis, it alone cannot justify the existence of the compounds especially when an extract contains a mixture of different molecules (Mak et al., 2013).

The GC-MS chromatographic analysis of the chloroform and ethyl acetate extracts revealed the presence of various bioactive compounds. The compounds like phenol 2,4-bis(1,1-dimethylethyl)-, 1,2-benzenedicarboxylic acid dibutyl ester, celidoniol deoxy, nonadecane, tetratetracontane, 2-methyloctacosane and pentadecane were present in both the extracts but in different quantities. The compounds phenol 2,4-bis(1,1-dimethylethyl)- is known for its antibacterial and antioxidant activity (Teresa et al., 2014; Manorenjitha et al., 2013); Pentadecane has been reported to bear antibacterial activity (Yogeshwari et al., 2012); 1,2-benzenedicarboxylic acid dibutyl ester is known to possess antibacterial, antifouling and pesticidal activity (Khatiwora et al., 2012; Jenecius et al., 2012; Wanxi et al., 2014; Nonadecane is antimicrobial and cytotoxic (Hsouna et al., 2011); 2,5-octadecanoic acid methyl ester is known for the antiviral, antibacterial and antioxidant nature (Linton et al., 2013; Sudharshan et al., 2010); 2-methyltetracosane is a free radical scavenger (Ramya et al., 2015); tetratetracontane is antibacterial (Gumgumjee and Hajar, 2015), tetracosane has shown cytotoxicity towards the gastric cancer cells by inducing apoptosis (Uddin et al., 2012); Celidoniol deoxy also known as nonacosane is antibacterial and anti-inflammatory (Kose et al., 2016; Zakaria et al., 2014). The biological activities of certain compounds like 3-Ethyl-3-methylheptane; 2-Cyclohexyl-3-isopropyl-pent4-en-2-ol; 2-Bromotetradecane; hexatriacontane; 1-chlorononadecane; 3-Phenoxypropylamine,2-allyl,beta,hydroxyl-N-[3.3-dimethylpropargyl]; 1-Heptanol,2,4-dimethyl-, (R,R)-(+)-; etc. have not been reported and hence marked as unknown. Based on the GC-MS studies, most of the chemical constituents appear as biologically active compounds and have shown to bear pharmacologic activities which may contribute to the therapeutic potential of the yeast. Apart from the chemical constituents with therapeutic potential certain compounds are found to help the insect in chemical communication. The compounds like Celidoniol deoxy is known to aid in chemical communication in the mosquito Anopheles stephensi and also act as a pheromone (Brei et al., 2004; Grant et al., 1987); nonadecane and 2-methyloctacosane and are the cuticular hydrocarbons of insects that also aids in chemical communication (Spikes et al., 2010; . Colazza et al., 2007). Docosane is report to aid in host egg parasitization that can be used as biocontrol agent (Paul et al., 2002). Christensen (2010) has reported that the insect associated yeasts produce volatile compounds for intraspecific and interspecific communication in insects.

The yeast extracts has shown potent antimicrobial activity against the human pathogens used and hence proves its antimicrobial nature. The chloroform extract showed higher antimicrobial activity as compared to the ethyl acetate extract which may be attributed to the more number of bioactive chemical constituents in the chloroform extract as compared to the ethyl acetate extract evaluated by GC-MS analysis. Several research studies have previously reported the antimicrobial nature of yeasts especially in biopreservation of food and yeasts as biocontrol agents (Younis et al., 2017; Mewa-Ngongang et al., 2017; Knight and Witzgall, 2013) but the antimicrobial nature of yeasts in light of its chemical constituents has not been given much importance. Thus this study reports the probability of antimicrobial nature of the yeast isolated from insect gut with regards to its bioactive chemical constituents.

\section{Conclusion:-}

Insects display a diversity of microbial association owing to the diverse nature of insects on earth. These insect associated microbes act as a large reservoir of bioactive natural products that can be used in human therapy. The yeast Cryptococcus rajasthanensis reported in this study has been isolated from the insect Bombyx mori gut and has shown considerable antimicrobial activity against the pathogenic microbial forms. The GC-MS study has also analyzed diverse chemical constituents that are known to be biologically active. Thus, the study shows the bioactive potency of the yeast isolated from the insect gut. It also indicates that the yeasts associated with the insect gut act as the reservoir for bioactive natural products that can be explored for human therapy. However, this study needs to further purify and evaluate the main chemical constituents that play the biologically active role and also to explore the novel bioactive compounds from the insect-gut microflora.

\section{Acknowledgments:-}

Delicia Avilla Barretto is grateful to Department of Science \& Technology- Innovation in Science Pursuit for Inspired Research (DST-INSPIRE) with (Award letter number: DST/INSPIRE FELLOWSHIP/2013/349 Dated: 16-08-2013) for financial assistance to complete this research work. The authors are thankful to the technical staff of 
University Scientific and Instruments Centre (USIC), Karnatak University Dharwad for their technical support and to the Kerala Forest Research Institute (KFRI), Peechi, Thrissur, Kerala, India for the GC-MS analysis. The authors also thank Eurofins Genomics India Pvt. Ltd., Bangalore, India for the yeast strain identification.

\section{Conflict of interest:-}

Authors do not have any conflict of interest related to the manuscript.

\section{References:-}

1. Abubacker, M.N. and Devi, P.K. (2015): In vitro Antifungal Potentials of Bioactive Compounds Heptadecane, 9- hexyl and Ethyl iso-allocholate isolated from Lepidagathis cristata Willd. (Acanthaceae) leaf. Br Med Bull., 336-341.

2. Beemelmanns, C., Guo, H., Rischer, M. and Poulsen, M. (2016): Natural products from microbes associated with insects. Beilstein J Org Chem., 12:314-327.

3. Bode, H.B. (2011): Chapter 5: Insect associated microorganisms as a source for novel Secondary metabolites with therapeutic potential. In: Vilcinskas A. (eds) Insect Biotechnology. Biologically-Inspired Systems, vol 2. Springer, Dordrecht. p.78.

4. Bonev, B., Hooper, J. and Parisot, J. (2008): Principles of assessing bacterial susceptibility to antibiotics using the agar diffusion method. J Antimicrob Chemother., 61: 1295-1301.

5. Bottone, E.J. (1980): Cryptococcus neoformans: Pitfalls in Diagnosis Through Evaluation of Gram-Stained Smears of Purulent Exudates. J Clin Microbiol., 12(6): 790-791

6. Brachmann, A.O. and Bode, H.B. (2013): Identification and bioanalysis of natural products from insect symbionts and pathogens. Adv Biochem Eng Biotechnol., 135:123-55.

7. Brei, B., Edman, J.D., Gerade, B. and Clark, J.M. (2004): Relative Abundance of Two Cuticular Hydrocarbons Indicates Whether a Mosquito Is Old Enough to Transmit Malaria Parasites. J. Med. Entomol., 41(4): 807-809.

8. Calderon-Cortes, N., Quesada, M., Watanabe, H., Cano-Camacho, H., Oyama, K. (2012): Endogenous plant cell wall digestion: a key mechanism in insect evolution. Annu Rev Ecol Evol Syst., 43:45-71.

9. Chapman, A.D.A. (2007): Report for the Department of the Environment and heritage, September 2005. Australian Biodiversity Information Services, Toowoomba, Australia. www.environment.gov.au/biodiversty/abrs/publocations/other/species-numbers/03-02-groupsinvertebrates.html

10. Christensen, T.A. (2010): Methods in insect sensory neuroscience. CRC press publishers. ISBN 9781420039429

11. Cita, Y.P., Suhermanto, A., Radjasa, O.K. and Sudharmono, P. (2017): Antibacterial activity of marine bacteria isolated from sponge Xestospongia testudinaria from Sorong, Papua. Asian Pac J Trop Biomed., 7(5): $450-454$.

12. Colazza, S., Aquila, G., De Pasquale, C., Peri and Millar, J.G. (2007): The Egg Parasitoid Trissolcus basalis uses n-nonadecane, a Cuticular Hydrocarbon from its Stink Bug Host Nezara viridula, to Discriminate Between Female and Male Hosts. Chem Ecol., 33:1405-1420

13. Davis, T.S., Hofstetter, R.W., Foster, J.T., Foote, N.E. and Keim, P. (2011): Interactions between the yeast Ogataea pini and filamentous fungi associated with the western pine beetle. Microb Ecol., 61(3):626-34.

14. Dillon, R.J., Dillon, V.M. (2004:) The gut bacteria of insects: nonpathogenic interactions. Annu Rev Entomol., 49:71-92.

15. Douglas, A.E. (2015): Multiorganismal insects: diversity and function of resident microorganisms. Annu Rev Entomol., 7 (60): 17-34.

16. Douglas, A.E., Minto, L.B., Wilkinson, T.L. (2001): Quantifying nutrient production by the microbial symbionts in an aphid. J Exp Biol., 204:349-58.

17. Engel, P. and Moran, N.A. (2013): The gut microbiota of insects - diversity in structure and function. FEMS Microbiol Rev., 37: 699-735

18. Ezenwa, V.O., Gerardo, N.M., Inouye, D.W., Medina, M., Xavier, J.B. (2012): Microbiology: animal behavior and the microbiome. Science., 338:198-99.

19. Gibson, C.M. and Hunter, M.S. (2010): Extraordinarily widespread and fantastically complex: comparative biology of endosymbiotic bacterial and fungal mutualists of insect. Ecol let., 13(2): 223-234.

20. Gonzalez, F. (2014): Symbiosis between yeasts and insects. Introductory Pap Fac Landsc Archit, Hortic Crop Prod Sci., 3:1-52.

21. Grant, G.G., Frech, D., MacDonald, L., Slessor, K.N. and King, G.G.S. (1987): Copulation releaser pheromone in body scales of female whitemarked tussock moth, Orgyia leucostigma (Lepidoptera: Lymantriidae): identification and behavioral role. J Chem Ecol., 13:345-356. 
22. Gumgumjee, N.M. and Hajar, S.A. (2015): Antibacterial activities and GC-MS analysis of phytocomponents of Ehretia abyssinica R.Br. ex fresen. Int J Appl Biol Pharm Tech.,6(2): 236-241.

23. Hsouna, A.B., Trigui, M., Mansour, R.B., Jarraya, R.M., Damak, M., Jaoua, S. (2011): Chemical composition, cytotoxicity effect and antimicrobial activity of Ceratonia siliqua essential oil with preservative effects against Listeria inoculated in minced beef meat. Int J Food Microbiol., 148: 66-72.

24. Jenecius, A.A., Uthayakumari, F. and Mohan, V.R. (2012):GC-MS determination of bioactive components of Sauropus bacciformis blume (euphorbiaceae) J Curr Chem Pharm Sc.,2(4): 347-358.

25. Khatiwora, E., Adsul, V.B., Kulkarni, M., Deshpande, N.R. and Kashalkar, R.V. (2012): Antibacterial activity of Dibutyl Phthalate : A secondary metabolite isolated from Ipomoea carnea stem. J Pharm Res., 5(1): 150-152.

26. Knight, A.L. and Witzgall, P. (2013): Combining mutualistic yeast and pathogenic virus--a novel method for codling moth control. J Chem Ecol., 39(7):1019-26.

27. Köse, Y.B., Iscan, G. and Demirci, B. (2016): Antimicrobial Activity of the Essential Oils Obtained from Flowering Aerial Parts of Centaurea lycopifolia Boiss. et Kotschy and Centaurea cheirolopha (Fenzl) Wagenitz from Turkey. J Essent Oil Bear Pl., 19 (3): 762 - 768.

28. Limtong, S., Kaewwichian, R., Yongmanitchai, W. and Kawasaki, H. (2014): Diversity of culturable yeasts in phylloplane of sugarcane in Thailand and their capability to produce indole-3-acetic acid. World J Microbiol Biotechnol., 30: 1785-1796.

29. Linton, R.E.A., Jerah, Lihan, S., Ahmad, I.B. (2013): The Effect Of Combination Of Octadecanoic Acid, Methyl Ester And Ribavirin Against Measles Virus. Int J Sci Tech Res., 2(10): 181-184.

30. Lockner, D. (1979): Treatment of refractory anemias with methenolone. Acta Med Scand., 205(12): 97-101.

31. Mak, Y.W., Chuah, L.O., Ahmad, R. and Bha, R. (2013): Antioxidant and antibacterial activities of hibiscus (Hibiscus rosa-sinensis L.) and Cassia (Senna bicapsularis L.) flower extracts. J King Saud Univ - Science., 25: 275-282.

32. Manorenjitha, M.S., Norita, A.K., Norhisham, S., Asmawi, M.Z. (2013): GC-MS analysis of bioactive components of Ficus seligiosa (Linn) Stem. Int J Pharm Biol Sci., 4: 99- 103.

33. Mewa-Ngongang, M., Ntwampe, S.K.O., du Plessis, H.W., Mekuto, L. and Jolly, N.P. (2017): Biopreservatives from yeasts with antimicrobial activity against common food, agricultural produce and beverage spoilage organisms Antimicrobial research: Novel bioknowledge and educational programs (A. Méndez-Vilas, ed.) 219228.

34. Nasir, H. and Noda, H. (2003): Yeast-like symbiotes as a sterol source in anobiid beetles (Coleoptera, Anobiidae): possible metabolic pathways from fungal sterols to 7-dehydrocholesterol. Arch Insect Biochem Physiol., 52:175-82.

35. Paul, A.V.N., Singh, S. and Singh, A.K. (2002): Kairomonal effect of some saturated hydrocarbons on the egg parasitoids, Trichogramma brasiliensis (Ashmead) and Trichogramma exiguum (Hymenoptera: Trichogrammatidae). J Appl Entomol., 126: 409-416.

36. Poojary, M.M., Vishnumurthy, K.A., Adhikari, A.V. (2015): Extraction, characterization andbiological studies of phytochemicals from Mammea suriga. Journal of Pharmaceutical Analysis., 5(3):182-189.

37. Pozo, M.I., Lachance, M.A. and Herrera, C.M. (2012): Nectar yeasts of two southern Spanish plants: the roles of immigration and physiological traits in community assembly. FEMS Microbiol Ecol., 80(2): 281-293.

38. Rajagopal, R. (2009): Benefi cial interactions between insects and gut bacteria. Indian J Microbiol., 49:114119.

39. Ramya, B., Malarvili, T. and Velavan, S. (2015): GC-MS analysis of bioactive compounds in Bryonopsis laciniosa fruit extract. Int J Pharm Sci Res., 6(8): 3375-3379.

40. Saluja, P. and Prasad, G.S. (2007): Cryptococcus rajasthanensis sp. nov., an anamorphic yeast species related to Cryptococcus laurentii, isolated from Rajasthan, India. Int J Syst Evol Microbiol., 57: 414-418.

41. Sivaraman, T., Sreedevi, N.S. and Meenatchisundaram, S. (2017): Phytochemical and gas chromatographicmass spectrometric analysis of ethanolic extract of Rauvolfia serpentina root. Eur J Pharm Med Res., 4(2): 646650.

42. Sivasubramanian, R. and Brindha, P. (2013): In- vitro cytotoxic, antioxidant and GC-MS studies on Centratherum punctatum cass. Int J Pharm Sci., 5( 3): 364-367.

43. Soria-Mercado, I.E., Villarreal-Gómez, L.J., Rivas, G.G., Nahara, E. and Sánchez, A. (2012): Chapter 3: Bioactive Compounds from Bacteria Associated to Marine Algae. InTech publishers. ISBN 978-953-51-0151-2.

44. Spikes, A.E., Paschen, M.A. Millar, J.G., Moreira, J., Hamel, P.B., Schiff, N.M. and Ginzel, M.D. (2010): First Contact Pheromone Identified for a Longhorned Beetle (Coleoptera: Cerambycidae) in the Subfamily Prioninae. J Chem Ecol., 36:943-954. 
45. Sudharsan, S., Saravanan, R., Shanmugam, A., Vairamani, S., Mohan kumar, R., Menaga, S. and Ramesh, N. (2011): Isolation and Characterization of Octadecanoic Acid from The Ethyl Acetate Root Extract of Trigonella foneum graecum L. by Using Hydroponics Method. J Bioterr Biodef., 2:-1-5.

46. Tamura, K., Dudley, J., Nei, M. and Kumar, S. (2007): MEGA4: Molecular Evolutionary Genetics Analysis (MEGA) software version 4.0. Mol Biol Evol.,24:1596-1599.

47. Tejado, A., Penaa, C., Labidia, J., Echeverria, J.M. and Mondragon, I. (2007): Physico-chemical characterization of lignins from different sources for use in phenol-formaldehyde resin synthesis. Bioresource Technology., 98: 1655-1663.

48. Teresa, R.C.M., Rosaura, V.G., Elda, C.M. and Ernest, G.P. (2014): The avocado defense compound phenol2,4-bis (1,1-dimethylethyl) is induced by arachidonic acid and acts via the inhibition of hydrogen peroxide production by pathogens. Physiol Mol Plant Pathol., 87: 32-41.

49. Uddin, S.J., Grice, D., and Tiralongo, E. (2012): Evaluation of cytotoxic activity of patriscabratine, tetracosane and various flavonoids isolated from the Bangladeshi medicinal plant Acrostichum aureum . Pharmaceutical Biology., 50(10): 1276-1280.

50. Urubschurov, V. and Janczyk, P. (2011): Biodiversity of Yeasts in the Gastrointestinal Ecosystem with Emphasis on Its Importance for the Host. Edited by Oscar Grillo and Gianfranco Venora, p. 277. ISBN 978953-307-772-7

51. Vega, F.E. and Dowd, P.F. (2005): The role of yeasts as insect endosymbionts. In: Vega FE, Blackwell M, editors. Insect-Fungal Associations: ecology and evolution. Oxford University Press; New York., pp. $211-243$.

52. Wanxi, P., Shengbo, G., Dongli, L. and Daochun, Q. (2014): Molecular characteristics of three extractives of Cinnamomum camphora leaves. Pak J Pharm Sci., 27.

53. Witzgall, P., Proffit, M., Rozpedowska, E., Becher, P.G., Andreadis, S., Coracini, M., Lindblom, T.U., Ream, L.J., Hagman, A., Bengtsson, M., Kurtzman, C.P., Piskur, J., Knight, A. (2012): "This is not an apple"-yeast mutualism in codling moth. J Chem Ecol., 38(8):949-57.

54. Yogeswari, S., Ramalakshmi, S., Neelavathy, R. and Muthumary, J. (2012): Identification and Comparative Studies of Different Volatile Fractions from Monochaetia kansensis by GCMS Global J Pharm., 6 (2): 65-71.

55. Younis, G., Awad, A., Dawod, R.E. and Yousef, N.E. (2017): Antimicrobial activity of yeasts against some pathogenic bacteria. Vet World., 10(8): 979-983.

56. Zakariaa, M.B., Vijayasekarana, Ilhama, Z. and Muhamad, N.A. (2014): Anti-Inflammatory Activity of Calophyllum inophyllum Fruits Extracts. Procedia Chemistry., 13:218 - 22 